\title{
Is the solar cycle still a puzzle?
}

\author{
Elena E. Benevolenskaya ${ }^{1,2} \dagger$ \\ ${ }^{1}$ W. W. Hansen Experimental Physics Laboratory, Stanford University, USA \\ email: elena@quake.stanford.edu \\ ${ }^{2}$ Pulkovo Astronomical Observatory, St. Petersburg, Russia
}

\begin{abstract}
The solar magnetic cycle affects all levels of the Sun including the convection zone, photosphere, chromosphere and corona. Recent advances in solar space missions (Yohkoh, SOHO and others) and, also, ground-based observations provide us an excellent opportunity to investigate solar magnetic activity in detail, and to draw a new picture of the solar magnetic cycle. Magnetic field appears on the solar surface as a result dynamo processes in the convection zone, and forms bipolar complexes of solar activity. These complexes can be seen in the photosphere as dark sunspots surrounded by the bright plages extended into chromosphere, with arcades of coronal loops best observed in EUV and soft X-rays. The coronal loops reflect the large-scale magnetic structure of complexes of activity. The new data reveal fundamental changes in the magnetic topology during the solar cycle, and details of the polar magnetic field reversals occurring near the sunspot maximum. The solar synoptic maps obtained from the photospheric and coronal data display a close correlation between the erupted magnetic flux and coronal emissions and show large-scale magnetic connectivities. The brief review of solar cycle studies is presented.
\end{abstract}

Keywords. solar cycle, solar magnetic field, coronal activity, etc.

\section{Introduction}

Is the solar cycle still a puzzle? How deep our understanding of the physical processes involved into the solar activity? These and many others questions arise when we are drawing the picture of the solar cycle. If we look at the solar activity in time (Figure 1) we find that the each 11-year sunspot cycle looks different. But we know that the solar activity is obeyed to the some laws or rules. The 22-years solar magnetic cycles form from pair of consecutive sunspot cycles and begin on the even sunspot cycles according to the Zürich numbering (Gnevyshev and Ohl, 1948). They manifest themselves in reversal of polarity of sunspots (Hale's law) from one 11-year cycle to another. During a solar sunspot cycle, the magnetic topology changes from largely poloidal field in the activity minima to predominantly toroidal field in the maxima. Magnetic polarity of the fields is reversed every 11 years. The toroidal field forms bipolar active regions and sunspots in low-latitude zones which migrate from approximately $30^{\circ}$ latitude towards the equator during the 11-year sunspot cycles (or magnetic half-cycles) forming the famous 'butterfly' diagram. It is assumed that the cyclic behavior of the solar magnetic field is associated with dynamo processes in the solar interior.

Here, I briefly represent only three, but very important aspects of the current solar cycle 23. Variations of the amplitude of the solar cycle and Gnevyshev-Ohl's rule, the polar magnetic field reversals and the axisymmetrical structure of the solar cycle in photosphere and corona. 

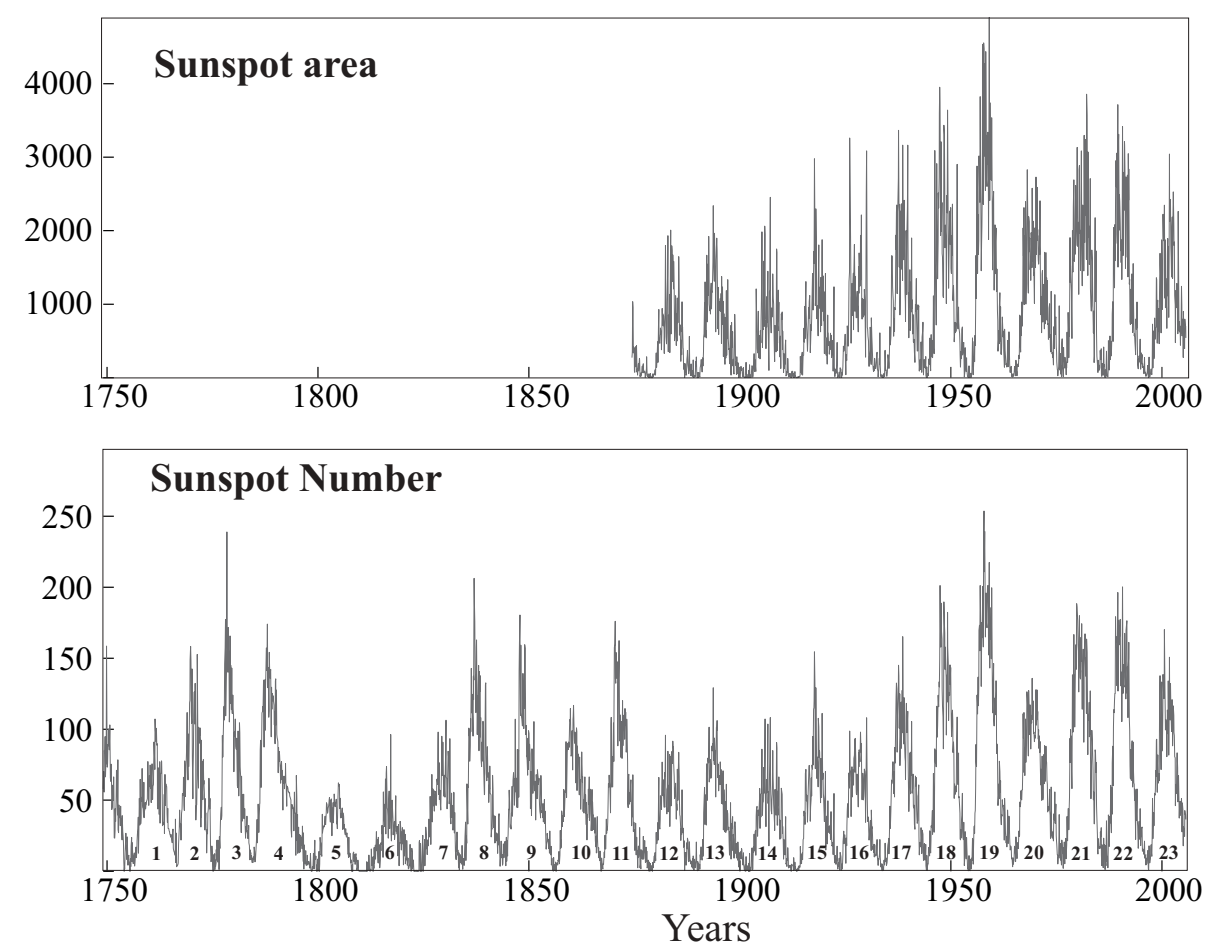

Figure 1. Plots of the sunspot area (upper panel) and sunspot number (bottom panel).

\section{Gnevyshev and Ohl's rule and solar magnetic cycle}

The Gnevyshev-Ohl's rule and its violation is timely problem. Now we are closely to the violation of this rule in pair of cycle 22-23. The rule, itself, tells that the 22 -year solar cycle begins on the even according to the Zurich numbering and the total number of annual averaged sunspot numbers during the odd-numbered sunspot cycle exceeds that of its preceding even-numbered sunspot cycle. The amplitude of odd cycle is mostly greater than the even cycle in the pair. Since cycle 12 , the ratio is about 1.4 , The violation of Gnevyshev-Ohl's (G-O) rule took place in the pair of cycles 4 and 5 for the cycles 2-21.

Figure $2 a, b$ displays the correlation between odd and even cycles in pairs even-odd and odd-even. We see practically no or weak correlation between odd and even cycles in pair odd-even(50\%). The cycles are highly correlated (91\%) between an even-numbered cycle and its following odd-numbered cycle.

The possible explanation of the existence or the violation of this rule was done by Pudovkin and Benevolenskaya (1985). It was suggested that the violation of the G-O rule is the consequence of the influence of the variations of the primodial magnetic field on the dynamo process in the convective zone. Durney (2000) proposed that the G-O rule may be a consequence of nonlinear interactions of velocity and magnetic field that provide the stabilizing mechanism. Dikpati et al. (2006) using dynamo transport model declare about the violation G-O for the next pair sunspot cycle. They predict the cycle 24 higher than 23. Komitov and Bonev (2000) predicted the violation of G-O rule in the pair of cycles 22 and 23. This was based on the high maximum annual average Wolf number for cycle 22 (157.6). The consequent development of cycle 23 confirms their conclusion. The present cycle is lower than the preceding cycle 22 as well as than the previous odd numbered cycles 19 and 21. Therefore, they argue that this behavior of cycle 23 might be a signal for an upcoming centurial solar minimum. They connected the violation with 


$$
\Sigma \mathbf{W}_{i+1}
$$

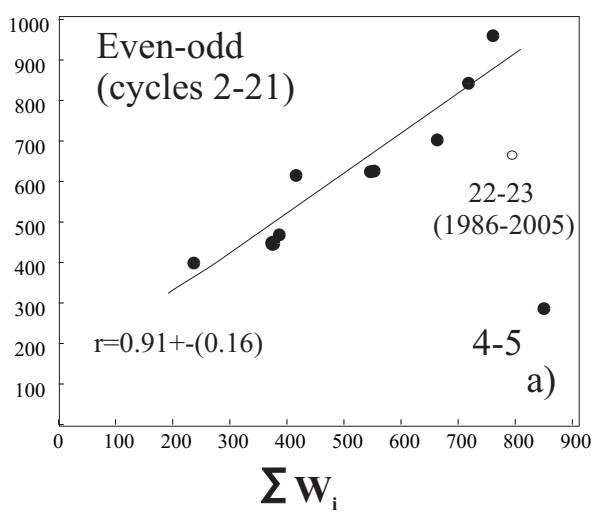

$\Sigma W_{\text {i-1 }}$

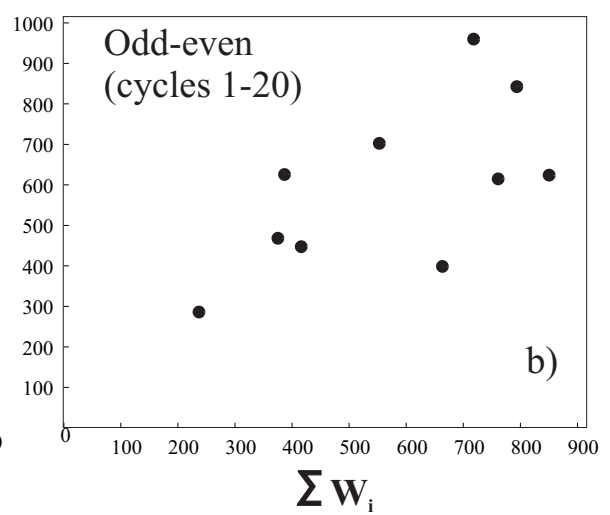

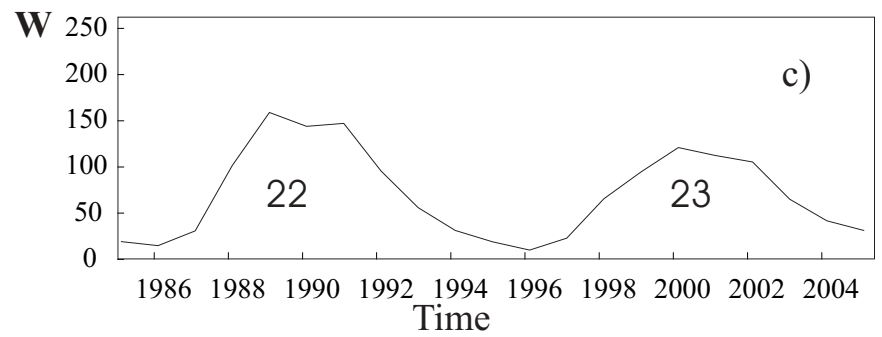

Figure 2. a) Scatter plot for total sunspot number during the odd cycle as function of total sunspot number in even cycle on Zurich numbering; b) Scatter plot for total sunspot number during the even cycle as function of total sunspot number in odd cycle on Zurich numbering; c)plot of annual sunspot number for cycles 22 and 23 .

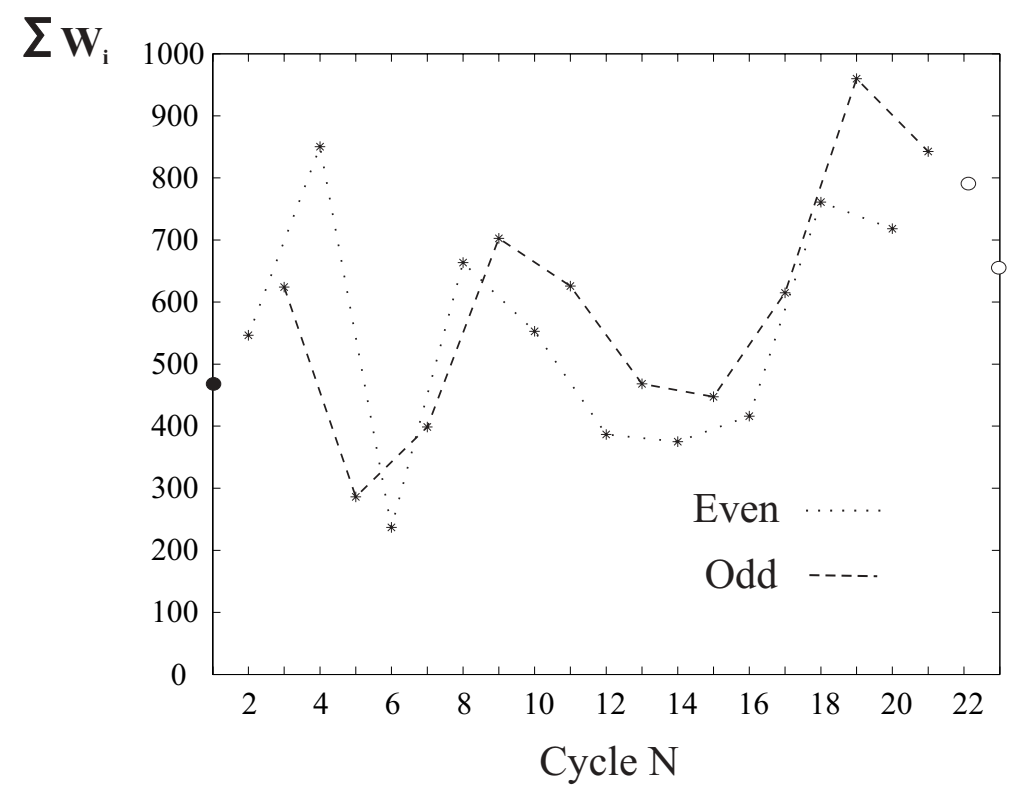

Figure 3. Plots for total sunspot numbers for even cycles(points lines) and odd cycles (dash lines). Cycles 22 and 23 is marked by circlesn and unpaired cycle 1 is marked by dark circle. 
declining phase of both the 100 or the 200 years solar cycle. This is in contradictory with the conclusion of Dikpati et al. that the next cycle will be higher than current. But in both predictions we have deal with the violation of Gnevyshev-Ohl's rule.

\section{Polar magnetic field reversals}

The solar magnetic field and, particularly, the polar magnetic field, is dominated by small magnetic elements of mixed polarities as has been shown by Severny (1971). Lin, Varsik, Zirin (1994) have studied high-resolution magnetograms of Big Bear Solar Observatory (BBSO) and confirmed that the fields are concentrated into small magnetic elements of both polarities. Although the polar regions may appear as unipolar in lowresolution magnetograms. Lin, Varsik and Zirin have estimated magnetic flux in latitude zones from $70^{\circ}$ to $80^{\circ}$ and found the magnetic elements of one polarity has a solar cycle dependence. This is also in agreement with the MDI data for higher latitudes (Figure 4b,c) in cycle 23 .

The polar magnetic filed reversals were discovered during the maxima of sunspot cycle 19 (Babcock and Livingston, 1958; Babcock, 1959). The polarity of the high-latitude dipolar magnetic field of the sun was opposite to that of the earth's from 1953 to 1957. About the middle of 1957 the polarity of the magnetic field near the south heliographic pole was reversed. The reversal of the field near the north pole was occurred in November, 1958. Then, the sun's polar field became parallel to that of the earth's.

The following reversals were observed in cycle 20. According to Waldmeier, Cycle 20 has shown an anomaly (Waldmeier, 1973). While the southern polar zone of prominences behaved regularly, two polar zones instead of one appeared in the northern hemisphere. The first was regular in every respect and reached the pole shortly after sunspot maximum. The second, irregular and weaker, appeared at mean latitudes only when the regular zone was already disappearing. This second zone drifted towards the pole and disappeared at the beginning of 1971. Compared to the first zone, the second had a delay of about 2 yr. Howard (1974) pointed out that the 'anomalous' filament motion found by Waldmeier in the north referred to a true field reversal. Later Makarov and Fatianov (1982) identified a three-fold magnetic field reversal in Cycle 20 using $H_{\alpha}$ synoptic charts. Other three-fold reversals of the solar polar magnetic field in one of the hemispheres are found to have happened the during last 120 yrs (Makarov and Sivaraman, 1989a, 1989b). These three-fold reversals took place in the southern hemisphere alone in cycles 12,14 and in the northern hemisphere alone in solar cycles 16, 18 and 20. Usually, the single reversals took place in the odd Cycles.

During the current solar cycle 23 the polar magnetic field reversals were single in both hemispheres. Figure 4 illustrates the process of the polar magnetic field reversals. The total unsigned magnetic flux of the radial magnetic field $F r=\left|F_{+}\right|+\left|F_{-}\right|$for polar caps $78^{\circ}-88^{\circ}$ is presented in Figure $4 a$ for the low-resolution MDI synoptic maps ( stepped in 10 in both the latitude and the longitude) with 'offset' correction (Benevolenskaya et al., 2006 in press). There is a N-S asymmetry in the distributions of the total polar magnetic flux for low-resolution maps: $\mathrm{Fr}=1.5-1.8 \times 10^{22} \mathrm{Mx}$ and $\mathrm{Fr}=2.0-2.5 \times 10^{22}$ Mx for the North and South polar caps, correspondingly, before CR 2007 (September 2003)(Benevolenskaya, 2004). After that time, the total magnetic flux displays a small increasing in the North and decreasing in the South. The positive $\left|F_{+}\right| / F_{r}$ part of the magnetic flux is plotted in Figures $4 b$. The total signed magnetic flux are shown in Figure $4 c$.

The time of reversals can be easily determined at $\left|F_{+}\right| / F_{r}=0.5$ or from the signed magnetic flux (Figure 4b,d). This was in CR1975 (April 2001) in the North and in 

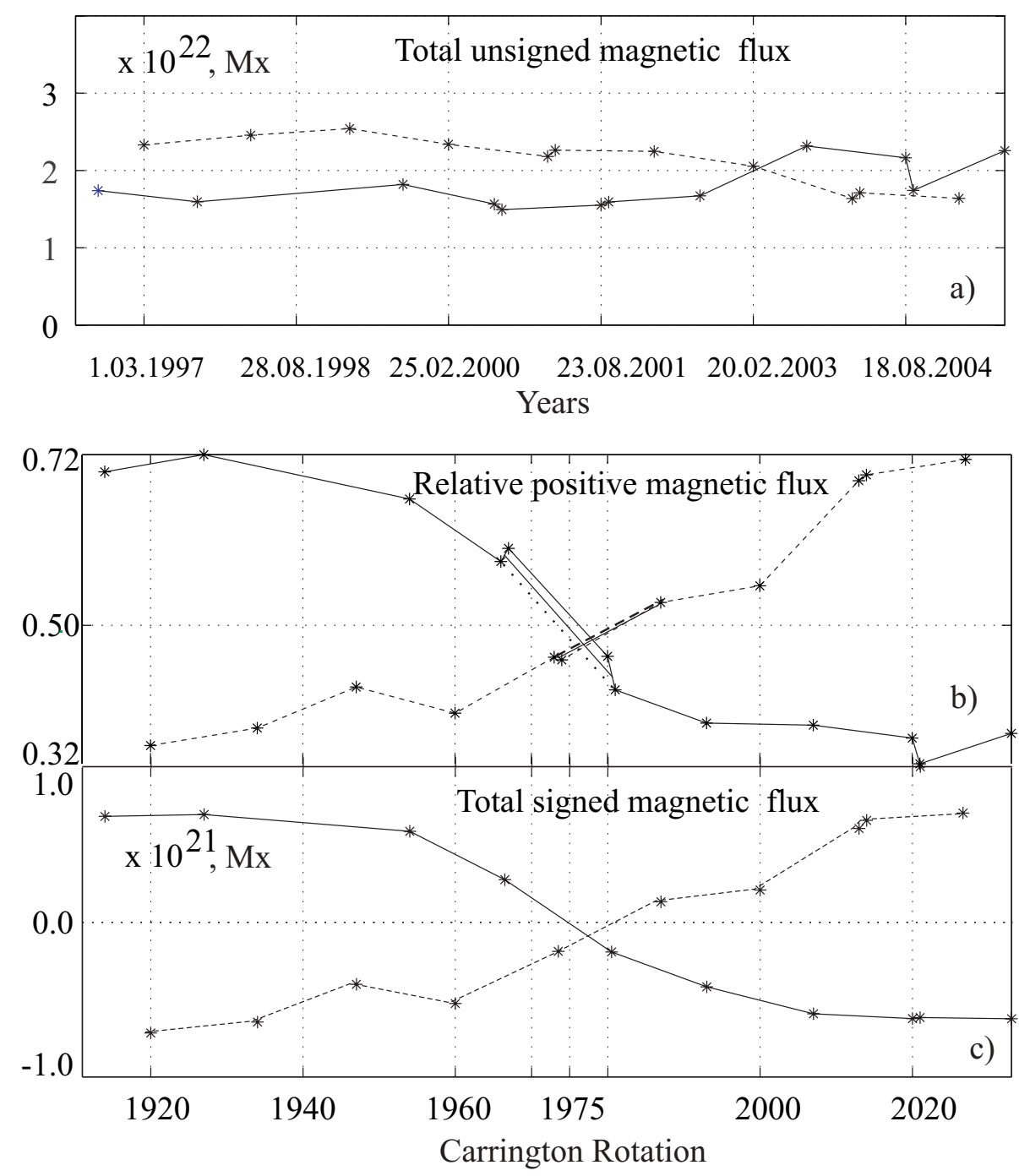

Figure 4. a) The total unsigned magnetic flux of the radial field component in the latitude zones from $78^{\circ}$ to $88^{\circ}$ in Northern (solid line) and Southern Hemispheres (dash lines); b) The relative positive polarity parts of magnetic flux in Northern (solid line) and Southern (dash line) hemispheres ; c) The total signed magnetic flux. The polar magnetic field reversal was in CR1975 \pm 2 (March 2001) in the North and in CR1980 \pm 2 (September 2001) in South.

CR1980 (August-September 2001) in South. This is close to the periods obtained by Durrant and Wilson (2003): CR1975 \pm 2 in North and CR1981 \pm 2 in South using the Kitt Peak synoptic maps.

\section{Solar magnetic cycle as the evolution of the axisymmetric magnetic magnetic field}

The studying of the EUV and soft X-ray corona (Benevolenskaya et al., 2001, 2002) reveals the coronal activity waves in the extreme ultraviolet data from SOHO/EIT (Delaboudiniere et al., 1995 )and soft X-rays from YOHKOH (Tsuneta et al., 1991) which are closely related to the magnetic activity. It is found, that the polar waves are represented 


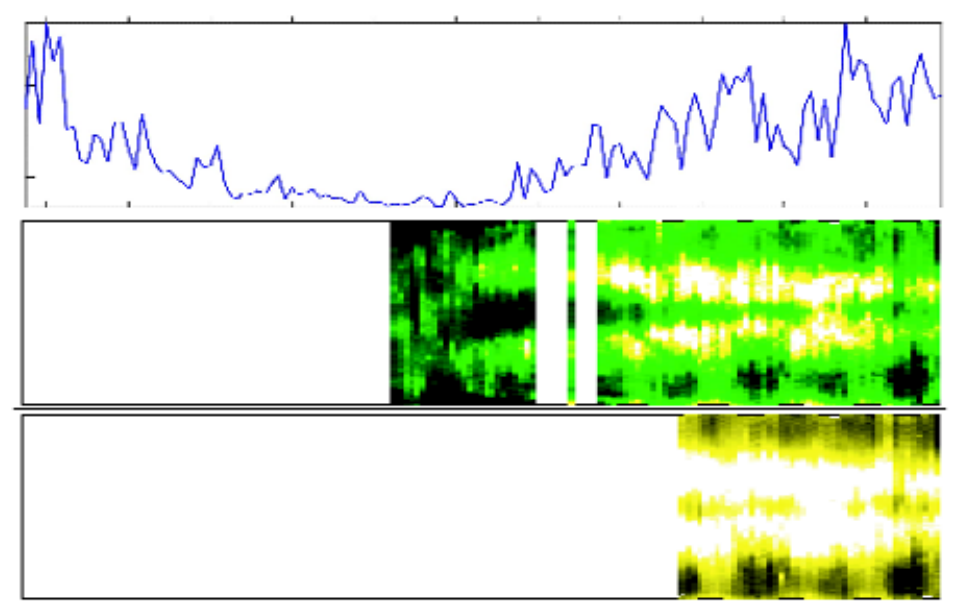

Figure 5. a) Sunspot area as a function of time from 11 November 1991 to 30 August 2003 (Carrington rotations from 1849 to 2006); Axisymmetrical distributions as a function of latitude from $-83^{\circ}$ to $83^{\circ}$ and time for: b)EUV flux in Fe XII and c) Fe XV lines (available only since CR1911, 28 June 1996); d) $B_{\|}$-component of the magnetic field, the gray-scale range is [-1G $1 \mathrm{G}]$; e) X-ray flux in the Al filter; f) X-ray flux in the AlMg filter; g) unsigned magnetic flux $\left|B_{\|}\right|$, in the $[020 \mathrm{G}]$ range.

by the bright coronal structures, which migrate to the poles during the rising phase of the solar cycle, which are formed by density enhancements in the poleward footpoints of giant loops in EUV emission. These loops can be considered as coronal proxy of a lines of magnetic field lines. These magnetic lines connect the magnetic fields of the following parts of active regions with the polar field. In other words, these giant magnetic loops connect the toroidal field of the new solar cycle with the polar poloidal field formed during the previous cycle, providing an important link between these two types of evolving magnetic topology (Benevolenskaya et al., 2001).

Indeed, the EIT time-latitude diagram (for example in line Fe XII, see Figure $5 b$ displays two sets of bright migrating structures in each hemisphere: low-latitude structures that migrate toward the equator following the evolution of $\left|B_{\|}\right|$(low-latitude coronal activity waves or 'butterfly' diagram) and high-latitude structures, or high-latitude waves, that migrate toward the poles parallel to the magnetic neutral lines. The high-latitude coronal structures are located $15-20^{\circ}$ higher in latitude than the neutral line. In the SXT axisymmetrical distributions (Figure $5 e, f$ ), the low-latitude migrating structures are similar to those in the EIT maps. However, the high-latitude structures look differently, without pronounced brightening in the polar regions, and are more uniform latitudinally, connecting the low-latitude bands with the polar regions. The 'impulses of solar activity', and, in particular, the 'coronal impulses' found in the EIT and SXT data are closely related to long-living complexes of solar activity with arcades of coronal loops (Benevolenskaya, 2003). At the beginning of the solar cycle, the magnetic flux appears at the photosphere in the form of bipolar magnetic complexes of activity at the middle latitudes as a sequence of impulses of solar activity. In the corona, we observe the magnetic loops connecting regions of opposite polarity inside the bipolar regions, and, in addition, giant loops connecting the following parts of the complexes of activity with the polar regions. Some portion of the erupted magnetic flux energy from the interior contributes to plasma heating in the coronal loops to $2-3 \times 10^{6}$. 
During the rising phase of the solar cycle, the footpoints of the giant loops in polarward become located closer and closer to the poles following the migrating zonal magnetic neutral lines. When the zonal neutral lines reach the poles then the polar magnetic field changes the polarity. After the polar magnetic field reversals the situation changes. The polar magnetic field has now the same polarity as the following parts of the bi-polar complexes of solar activity.

The connectivity from the following part of complexes of solar activity to the higher latitude zones becomes more random, and is mostly associated with small-scale regions of the opposite polarity, which are persistent over the solar disk together with the largescale magnetic field. Complexes of solar activity during the declining phase of the solar cycle are located more close to the solar equator; and, transequatorial loops connecting the complexes across the equator predominately appear in the declining phase.

\section{Conclusions}

- The violation of G-O rule takes place in the current magnetic cycle 22-23. The question is open if this is a result of interaction of secular cycles with hale cycle or influence of 'seed' magnetic field.

- The polar magnetic field reversal was in CR1975 \pm 2 (March 2001) in the North and in CR1980 \pm 2 (September 2001) in South according to the SOHO/MDI data.

- The solar cycle studies reveal a close relation of the coronal X-ray and the EUV emissions to the photospheric magnetic field. The evolution of coronal structures in Xray and EUV can be considered as a proxy of the coronal magnetic field and demonstrates a development of the solar magnetic cycle in corona.

\section{References}

Babcock, H. W., Livingston W. C., 1958, Science, 127, 1058

Babcock, H. D. 1959, ApJ, 130, 364

Benevolenskaya, E. E., Kosovichev, A. G., Scherrer, P. H. 2001, ApJ, 554, L107

Benevolenskaya, E. E., Kosovichev, A. G., Lemen, J. R., Slater G. L., Scherrer, P. H. 2002, ApJ, $571, \mathrm{~L} 181$

Benevolenskaya, E. E. 2003, Solar Phys., 216, 325

Benevolenskaya, E. E. 2004, Astron. Astrophys., 428, L5

Benevolenskaya, E. E., Hoeksema, J. T., Kosovichev, A. G., Liu, Y., Zhao, X., Scherrer, P.H. 2004, ApJ, in preparation

Dikpati, M., de Toma, G., Giman, P. 2006, GRL, 33(5), L05102

Durney B. 2000, Solar Phys, 196, 421

Durrant, C. J. and Wilson, P. R. 2003, Solar Phys., 214, 23

Gnevyshev, M.N., and Ohl, A.I. 1948, Astron. Zh(in russian), 35, 18

Howard, R. 1974, Solar Phys., 38, 59

Komitov, B., Bonev, B. Bulletin of the American Astronomical Society 2000, 32, 832

Lin, H., Varsik, J., Zirin, H. 1994, Solar Phys. 155, 243

Makarov, V. I. and Fatianov, M. P. 1982, Sov.Astron.Lett., 8, 631

Makarov, V. I. and Sivaraman, K. R. 1989a, Solar Phys., 123, 367

Makarov, V. I. and Sivaraman, K. R. 1989b, Solar Phys., 119, 35

Pudovkin M. I., Benevolenskaya, E.E. 1985 Solar Phys., 95, 381

Severny A. B. 1971, Q.Jl R. astr. Soc., 12, 363

Waldmeier, M. 1973, Solar Phys., 28, 389 